\title{
molecules
}

ISSN 1420-3049

(C) 2000 by MDPI

http://www.mdpi.org

\section{Synthesis of Potassium (1,1-Dioxothiolan-3-yl)-dithiocarbamate}

\author{
A.N. Vasiliev $^{1} *$ and A.D. Polackov ${ }^{2}$ \\ ${ }^{1}$ National Technological University, CITeQ, C.C.17, Cordoba 5016, Argentina \\ Tel.: 54-351-469-0585, Fax: 54-351-469-0585, E-mail: a.vasiliev@usa.net \\ ${ }^{2}$ Institute of Bioorganic Chemistry \& Petrochemistry, P.O.Box 305, Kiev 252034, Ukraine \\ Tel.: 380-44-261-4324
}

Received: 7 March 2000; revised form 10 August 2000/Accepted: 11 August 2000/Published: 15 August 2000

\begin{abstract}
A method of synthesizing bioactive potassium (1,1-dioxothiolan-3-yl) dithiocarbamate in high yield and purity is reported.
\end{abstract}

Keywords: dithiocarbamate, pesticide, catalysis.

\section{Introduction}

Metal salts of heterocyclic dithiocarbamates (DTC) are of potential interest in applications such as pesticides and antioxidants. In particular, potassium (1,1-dioxothiolan-3-yl)-dithiocarbamate is an effective fungicide with selective action [1,2]. The synthesis of this compound is difficult because of solvent effects and low solubility of some reagents in the appropriate solvents. As it was earlier supposed by Castro [3], the reaction between amines and carbon disulfide involves catalysis by strong base. Some preliminary investigations were devoted to investigation of mechanism of the reaction and effect of solvent $[4,5]$. This paper presents a part of our work on synthesis optimization of this salt.

\section{Results and Discussion}

The main synthetic route to dithiocarbamates is based on the interaction between the corresponding amine and $\mathrm{CS}_{2}$ in the presence of strong base (Scheme 1). In our work we used 3-aminothiolane as starting material.<smiles>NC1CCS(=O)O1</smiles>

$+\mathrm{CS}_{2}+\mathrm{C}_{2} \mathrm{H}_{5} \mathrm{O}^{-}$<smiles>O[C@@H]1[CH]CCC1</smiles><smiles>S=C([S-])NC1CCOC1</smiles> 
It was found that this method has some limitations. As it was shown earlier in our studies of the solvent effect, upon decreasing of the protoning ability of the solvent, the rate of dithiocarbamate formation increases [6]. In aprotic systems, the rate of the process increases with the increase of the dielectric permitivity of the solvent. However, the solubility of $\mathrm{KOH}$ in aprotic solvents is very low. Some of the solvents also possess low stability in alkaline media. Additionally, destruction of $\mathrm{C}_{4} \mathrm{H}_{7} \mathrm{SO}_{2} \mathrm{NH}_{2}$ and its transformation into the corresponding isocyanate can take place. So, the problem of solvent selection is critical in the synthesis. Use of water-dioxane mixture did not provide satisfactory yields and purity of the product. Better results were obtained by carrying out the reaction in ethanol or its mixture with DMF.

For optimization of the synthesis we studied the reaction mechanism. This investigation was concerned with the clarification of the claimed role of base catalysis in the process of amine addition to $\mathrm{CS}_{2}$ in ethanol media [3]. As it was supposed, the following reactions are possible in this process:

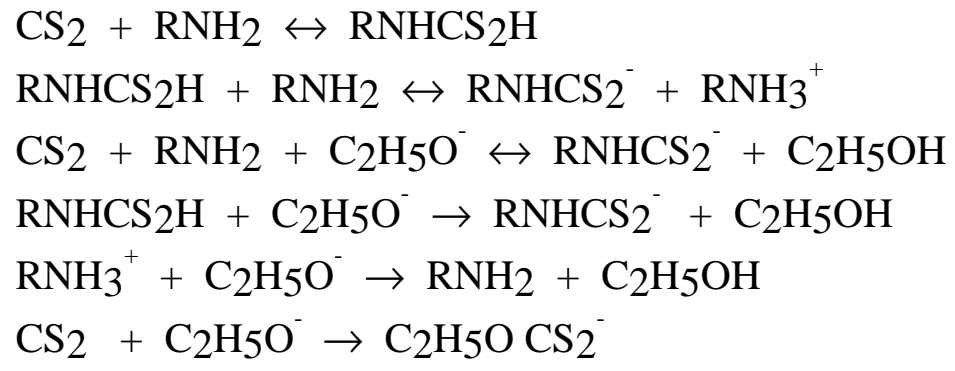

In our studies [7] both highly reactive methylamine and 1,1-dioxothiolan-3-yl-amine with lower amino group reactivity were used as model amines and benzylamine and piperidine were considered as reference materials. For all tested amines, the yield of dithiocarbamates corresponded to twice the amount of consumed amines in the absence of free ethylate ions. This is possible only if the reaction proceeds by routes (1) and (2) while route (3) does not take place. The kinetic data obtained [4,5] show that the effect of $\mathrm{C}_{2} \mathrm{H}_{5} \mathrm{O}^{-}$ion concentration on the total rate of dithiocarbamate accumulation is far smaller than expected if base catalysis (3) is the sole pathway. For 1,1-dioxothiolan-3-yl-amine as well as benzylamine the mentioned effect has near-zero value, while for piperidine the reaction rate increases 3-5 times when the concentration of $\mathrm{C}_{2} \mathrm{H}_{5} \mathrm{O}^{-}$increases by a factor of $10^{8}$ (addition of potassium ethylate). This fact provides us with evidence that the direct base catalyzed reaction makes no essential contribution to dithiocarbamate formation rate, even in the initial phase of the process, and unambiguously confirms the kinetic insignificance of this mechanism at quasi-equilibrium.

Selectivity of the reaction is determined by competition of routes (1) and (6) that requires an excess of amine to ethylate. For suppression of the reaction (2) an excess of $\mathrm{CS}_{2}$ is necessary. So, at the time of synthesis the reagents concentrations should satisfy the condition: $\left[\mathrm{CS}_{2}\right] \gg$ > $\left[\mathrm{RNH}_{2}\right]>\left[\mathrm{C}_{2} \mathrm{H}_{5} \mathrm{O}^{\circ}\right]$. Considering this condition, an original method of potassium (1,1-dioxothiolan-3-yl)-dithiocarbamate synthesis was developed. In accordance with this method, solutions of 3-amino-thiolane and $\mathrm{KOH}$ are simultaneously added to solution of $\mathrm{CS}_{2}$ in ethanol. Final product precipitates as white crystals.

\section{Conclusions}

An effective method of preparing potassium (1,1-dioxothiolan-3-yl)-dithiocarbamate was developed and optimized. The investigation showed that in this reaction catalysis by ethylate ion claimed by Castro does not take place. 


\section{Experimental}

\section{General}

2-Sulfolene (1) and 3-sulfolene (2) were analysed by GLC on a Hewlett Packard 5732A GC equipped with FID. Analysis conditions: 1,2 m x $3 \mathrm{~mm}$ column; mobile phase: 4,3\% of Carbowax 1500 on Gat chrome (80/100 mesh); column temperature: $130^{\circ} \mathrm{C}$; carrier gas, helium $(85 \mathrm{~mL} / \mathrm{min}$.); internal standard, sulfolane (3). $\mathrm{R}_{\mathrm{t}}$ (min.): $\mathbf{1}, 26,5 ; \mathbf{2}, 10,5 ; \mathbf{3}, 11,7$. The contents of $\mathbf{1}$ and bis-(1,1-dioxothiolan3 -yl)amine in the crude product was determined by potentiometric titration using $\mathrm{HClO}_{4}$ in $\mathrm{AcOH}$. The concentration of potassium (1,1-dioxothiolan-3-yl)-dithiocarbamate (5) was determined by consecutive titration of the product by $\mathrm{HCl}$ to neutralize the reaction mixture, followed by iodometric titration. ${ }^{1} \mathrm{H}$ NMR spectra were recorded on a Bruker-200X. instrument. IR spectra were recorded on a Jasco FT/IR-5300 spectrometer.

\section{3-Aminothiolane}

Compound $2(1 \mathrm{~kg}), 25 \% \mathrm{NH}_{4} \mathrm{OH}(3 \mathrm{~L})$ and $\mathrm{CaO}(30-50 \mathrm{~g})$ were loaded into a $5 \mathrm{~L}$ reactor. The mixture was stirred while the temperature was slowly rised to $80^{\circ} \mathrm{C}$ and maintained for $8 \mathrm{~h}$. Then the reaction mixture was cooled, treated with charcoal, filtered and the solvent was evaporated at reduced pressure. The resulting oily product contained $>90 \%$ of 3 -aminothiolane (4). For purification, the crude product was taken up in acidic water $(\mathrm{pH}=4-5)$ and the resulting solution was refluxed in the presence of charcoal, cooled, filtered and the water was then removed under reduced pressure. The resulting solid 3-aminothiolane hydrochloride salt was washed with acetone, redissolved in water, neutralized with a solution of $\mathrm{Na}_{2} \mathrm{CO}_{3}$ and the resulting emulsion was extracted with $\mathrm{CHCl}_{3}$. The organic phase was dried over anhydrous $\mathrm{Na}_{2} \mathrm{SO}_{4}$ and the solvent was removed. The final product had a purity of almost $100 \%$.

\section{Potassium (1,1-dioxothiolan-3-yl)-dithiocarbamate}

To a solution of $\mathrm{CS}_{2}(0,692 \mathrm{~L})$ in ethanol $(1 \mathrm{~L})$, solutions of $\mathrm{KOH}(663 \mathrm{~g})$ in $96 \%$ ethanol $(3 \mathrm{~L})$ and compound $4(1,35 \mathrm{~kg})$ in $96 \%$ ethanol $(2 \mathrm{~L})$ were slowly added while the temperature was kept between $10-15^{\circ} \mathrm{C}$. Then the reaction mixture was stirred for $12 \mathrm{~h}$ at ambient temperature. The resulting white precipitate was filtered off, washed with ethanol and dried. The yield of 5 was $91 \%$, m.p. 196-200 ${ }^{\circ} \mathrm{C}$.

Spectral data: ${ }^{1} \mathrm{H}-\mathrm{NMR}\left(200.13 \mathrm{MHz}, \mathrm{DMSO}_{\mathrm{d}}\right) \delta$ : 2.35 (m, 2H, 4-CH $\mathrm{CH}_{2}, 2.98\left(\mathrm{~m}, 4 \mathrm{H}, 2,5-\mathrm{CH}_{2}\right)$, 5.07 (sextet, 1H, 3-CH), 8.36 (d, 1H, NH). IR (KBr) cm ${ }^{-1}: 3146,2970,1518,1321,1302,1122,958$, 815,761 .

\section{References and Notes}

1. Parkhomenko P.I., Vasiliev A.N. SCK - a new low toxic fungicide from the family of dithiocarbamates, Abstr. 9th Int. Congress of Pesticide Chemistry, London, 1998, Abstr. 1B-012.

2. Register of pesticides and agrochemicals permitted for use in Ukraine; Univest Marketing: Kiev, 1999; p. 75.

3. Castro, E.; Cortes, K.; Santos, J. Kinetics and mechanism of the reaction of carbon disulfide with 
piperidine in ethanol. J. Org. Chem. 1982, 47, 3774-3777.

4. Polackov, A.D.; Vasiliev, A.N. Optimization of the synthesis of potassium (1,1-dioxothiolan-3-yl) dithiocarbamate. Rus. Journ. Appl. Chem. 1995, 68, 1666-1668.

5. Shkaraputa, L.N. Kononov, A.V.; Polackov, A.D. Preparation of dithiocarbamates. Ukr. Chem. J. 1991, 9, 979-989.

6. Polackov, A.D. Modelling and optimization of potassium (1,1-dioxothiolan-3-yl)-dithiocarbamate synthesis. Ph.D. Thesis. Institute of Bioorganic Chemistry and Petrochemistry, Kiev, 1992.

7. Polackov, A.D.; Vasiliev, A.N. Investigation of base catalysis in dithiocarbamate formation, Abstr. $35^{\text {th }}$ Congr. IUPAC, Istanbul, 1995, Sec. 1-3, -p. 624.

Samples Availability: Available from MDPI.

(C) 2000 by MDPI (http://www.mdpi.org). 